\title{
Norm Additivity Conditions for Normal Linear Functionals on von Neumann Algebras
}

\author{
By \\ Masaharu KUSUDA* \\ §1. Introduction
}

Let $M$ be a von Neumann algebra and let $\varphi$ and $\psi$ be bounded linear functionals on $M$. We then have the norm inequality $\|\varphi+\psi\| \leq\|\varphi\|+\|\psi\|$. On the other hand, it is well-known that if $\varphi$ and $\psi$ are positive, then $\|\varphi+\psi\|=\|\varphi\|+\|\psi\|$. In general, however, such an equality does not necessarily holds if both $\varphi$ and $\psi$ are not positive. The purpose of this paper is to investigate when the norm equality $\|\varphi+\psi\|=\|\varphi\|+\|\psi\|$ holds for given normal linear functionals $\varphi$ and $\psi$. Then the fact to play an essential role is the following:

Let $M$ be a von Neumann algebra and let $\varphi$ be a normal linear functional on M. Then we have

$$
\varphi(\cdot)=|\varphi|(v \cdot), \quad|\varphi|(\cdot)=\varphi\left(v^{\prime} \cdot\right), \text { and }\|\varphi\|=\|\varphi\| \|
$$

for all partial isometries $v^{\prime}$ in $M$ satisfying that $\varphi\left(v^{\prime}\right)=\|\varphi\|$, where $|\varphi|$ denotes the absolute value of $\varphi$ (c.f. [1, Lemma 2.3]).

In connection with this fact, we may expect that the set of those elements $x$, in the unit ball of $M$, with $\varphi(x)=\|\varphi\|$ has nice information on norms and absolute values of normal linear functionals on $M$. In fact, by employing such a set, we will give necessary and sufficient conditions for a pair of normal linear functionals $\varphi$ and $\psi$ to satisfy that $\|\varphi+\psi\|=\|\varphi\|+\|\psi\|$ or that $|\varphi+\psi|=|\varphi|+|\psi|$ (Theorem 2.1).

\section{§2. Results}

Let $M$ be a von Neumann algebra and let $\varphi$ be a normal linear functional on $M$. By the polar decomposition of $\varphi$, we mean the following expression:

$$
\varphi(\cdot)=|\varphi|(v \cdot) \text { and }|\varphi|(\cdot)=\varphi\left(v^{\prime} \cdot\right)
$$

Communicated by H. Araki, November 9, 1994. Revised December 15, 1994.

1991 Mathematics Subject Classifications: 46L10.

* Department of Mathematics, Faculty of Engineering, Kansai University,

Yamate-cho 3-3-35, Suita, Osaka 564, Japan. 
for some partial isometry $v$ in $M$ and a uniquè positive linear functional $|\varphi|$ on $M$ which satisfies that

$$
\|\varphi\|=\|\varphi\| \text { and }|\varphi(x)|^{2} \leq\|\varphi\| \varphi \mid\left(x^{\prime} x\right)
$$

for all $x$ in $M$ (cf. [2, 3.6.7], [3, 1.14.4], or [4, III. 4.2]). Note that this condition (*) ensures the uniqueness of $|\varphi|$ (cf. [2, 3.6.7] or [4, III. 4.6]). More precisely, if a positive linear functional $\psi$ satisfies that

$$
\|\psi\|=\|\varphi\| \text { and }|\varphi(x)|^{2} \leq\|\varphi\| \psi(x x),
$$

then $\psi=|\varphi|^{1}$. In general, there is some freedom for the choice of $v$, as is seen from [1, Lemma 2.3]. However, if $v v^{\prime}$ is exactly equal to the support projection $s(|\varphi|)$ of $|\varphi|$, i.e., the smallest of all projections $p$ such that $|\varphi|(p \cdot)=|\varphi|(\cdot)$, then $v$ is uniquely determined (cf. [3, 1.14.4], [4, III. 4.6]).

Now we set

$$
M_{\varphi}=\{x \in M \mid\|x\| \leq 1, \varphi(x)=\|\varphi\|\},
$$

which is a non-empty and weakly compact face of the unit ball of $M$. Hence $M_{\varphi}$ contains a partial isometry (cf. $[2,1.4 .7],[3,1.6 .5],[4$, I.10.2]).

Positive linear functionals $\varphi$ and $\psi$ on a $C$-algebra always satisfy that $\|\varphi+\psi\|=\|\varphi\|+\|\psi\|$ and $\varphi+\psi$ is positive. These are generalized as follows.

Theorem 2.1. Let $M$ be a von Neumann algebra and let $\varphi$ and $\psi$ be normal linear functionals on $M$. Then the following conditions are equivalent:

(1) $M_{\varphi} \cap M_{\psi}$ is not empty.

(2) $|\varphi+\psi|=|\varphi|+|\psi|$.

(3) $\|\varphi+\psi\|=\|\varphi\|+\|\psi\|$.

(4) $M_{\varphi} \cap M_{\psi}=M_{\varphi+\psi}$.

Proof. We first show that $M_{\varphi} \cap M_{\psi} \subset M_{\varphi+\psi}$. Without loss of generality, we can assume that $M_{\varphi} \cap M_{\psi}$ is not empty. For any $x$ in $M_{\varphi} \cap M_{\psi}$, we have

$$
\|\varphi\|+\|\psi\|=\varphi(x)+\psi(x)=(\varphi+\psi)(x) \leq\|\varphi+\psi\| .
$$

Since this mean that $(\varphi+\psi)(x)=\|\varphi+\psi\|$, we see that $x \in M_{\varphi+\psi}$.

$(1) \Rightarrow(2)$. Let $v$ be a partial isometry in $M_{\varphi} \cap M_{\psi}$. Since $v \in M_{\varphi+\psi}$, it follows from [1, Lemma 2.3] that

$$
|\varphi+\psi|(\cdot)=(\varphi+\psi)(v \cdot)=\varphi(v \cdot)+\psi(v \cdot)=|\varphi|(\cdot)+|\psi|(\cdot) .
$$

$(2) \Rightarrow(3)$. Since $|\varphi+\psi|,|\varphi|$ and $|\psi|$ are positive linear functionals, we have

$$
\begin{aligned}
\|\varphi+\psi\| & =\|\varphi+\psi|\|=| \varphi+\psi \mid(1)=(|\varphi|+|\psi|)(1) \\
& =|\varphi|(1)+|\psi|(1)=\|\varphi|\|+\|| \psi \mid\|=\|\varphi\|+\|\psi\| .
\end{aligned}
$$

\footnotetext{
${ }^{1}$ As the definition of a polar decomposition, we adopt the (right) polar decomposition mentioned in $[2,3.6 .7]$ and our absolute value $|\varphi|$ means $\left|\varphi^{\prime}\right|$ in the sense of the (left) polar decomposition mentioned in [3.1.14.4] and [4, III. \$4].
} 
(3) $\Rightarrow(4)$. We have only to show that $M_{\varphi+\psi} \subset M_{\varphi} \cap M_{\psi}$. Take any $x$ from $M_{\varphi+\psi}$. We then have

$$
\varphi(x)+\psi(x)=(\varphi+\psi)(x)=\|\varphi+\psi\|=\|\varphi\|+\|\psi\| .
$$

Now denote by $\mathfrak{R} z$ the real part of a complex number $z$ and by $\mathfrak{I} z$ the imaginary part of $z$, respectively. Since $\|\varphi\|+\|\psi\|$ is a real number, it follows from the above equality that

$$
\mathfrak{R} \varphi(x)+\Re(x)=\Re(\varphi(x)+\psi(x))=\|\varphi\|+\|\psi\|
$$

Here remark that

$$
\|\omega\| \geq|\omega(x)| \geq|\Re \omega(x)|
$$

for every bounded linear functional $\omega$ on $M$. We thus have

$$
0 \leq\|\varphi\|-\Re \varphi(x)=\Re(x)-\|\psi\| \leq 0 .
$$

Hence we conclude that $\|\varphi\|=\Re \varphi(x)$ and $\|\psi\|=\Re \psi(x)$. This and the inequality $(* *)$ show that $\Im \varphi(x)=0$, i.e., $\|\varphi\|=\varphi(x)$. Similarly we obtain that $\|\psi\|=\psi(x)$. We thus see that $x \in M_{\varphi} \cap M_{\psi}$.

(4) $\Rightarrow(1)$. Since $M_{\varphi+\psi}$ is not empty, this implication is clear.

Q.E.D.

Here recall that positive linear functionals $\varphi$ and $\psi$ on a $C^{\prime}$-algebra are said to be orthogonal if $\|\varphi-\psi\|=\|\varphi\|+\|\psi\|$.

Corollary 2.2. Let $M$ be a von Neumann algebra and let $\varphi$ and $\psi$ be positive normal linear functionals on $M$. Then the following conditions are equivalent:

(1) $\varphi$ and $\psi$ are orthogonal.

(2) $M_{\varphi} \cap M_{-\psi}$ is not empty.

\section{References}

[1] Kusuda, M., Polar decompositions of bounded linear functionals on operator subalgebras, Proc. Amer. Math. Soc., 118 (1993), 839-843.

[2] Pedersen, G.K., $C^{*}$-Algebras and their Automorphism Groups, Academic Press, London, 1979.

[3] Sakai, S., C*-Algebras and $W^{*}$-Algebras, Springer-Verlag, New York, 1970.

[4] Takesaki, M., Theory of Operator Algebras I, Springer-Verlag, New York, 1979. 
